\title{
Bevacizumab for the Treatment of Radiation-Induced Cerebral Necrosis: A Systematic Review of the Literature
}

\author{
Durim Delishaj ${ }^{\mathrm{a}, \mathrm{c}}$, Stefano Ursino ${ }^{\mathrm{a}}$, Francesco Pasqualettia, Agostino Cristaudo ${ }^{\mathrm{a}}$, \\ Mirco Cosottini ${ }^{\mathrm{b}}$, Maria Grazia Fabrini ${ }^{\mathrm{a}}$, Fabiola Paiar ${ }^{\mathrm{a}}$
}

\begin{abstract}
Radiation necrosis ( $\mathrm{RN}$ ) of brain tissue is a serious late complication of brain irradiation and recently bevacizumab has been suggested as treatment option of RN. There is a lack of data in the literature regarding the effectiveness of bevacizumab for the treatment of RN. The purpose of this review was to perform a comprehensive analysis of all reported cases using bevacizumab for the treatment of brain RN. In September 2016, we performed a comprehensive literature search of the following electronic databases: PubMed, Web of Science, Scopus and Cochrane Library. The research for the review was conducted using a combination of the keywords "radiation necrosis", "radiotherapy" and "bevacizumab" alongside the fields comprising article title, abstract and keywords. Randomized trials, non-randomized trials, prospective studies, retrospective studies and single case reports were included in the review. Our research generated 21 studies and 125 cases where bevacizumab had been used for the treatment of RN. The median follow-up was 8 months and the most frequent bevacizumab dose used was 7.5 $\mathrm{mg} / \mathrm{kg}$ for 2 weeks with a median of four cycles. Low-dose bevacizumab resulted in effectiveness with improvement in both clinical and radiographic response. The median decrease in $\mathrm{T} 1$ contrast enhancement and in T2/FLAIR signal abnormality was $64 \%$ and $60 \%$, respectively. A reduction in steroidal therapy was observed in majority of patients treated. Based on the data of our review, bevacizumab appears to be a promising agent for the treatment of brain RN. Future prospective studies are required to evaluate the role of bevacizumab in $\mathrm{RN}$ and to define the optimal scheduling, dosage and duration of therapy.
\end{abstract}

Keywords: Bevacizumab; Brain metastases; Brain irradiation; Stereotactic radiotherapy

\section{Introduction}

With the introduction of new radiation techniques such as in-

\footnotetext{
Manuscript accepted for publication February 06, 2017

aDepartment of Radiotherapy, University Hospital of Pisa, Italy

${ }^{b}$ Neuroradiology Unit, University Hospital of Pisa, Italy

${ }^{\mathrm{c} C}$ Corresponding Author: Durim Delishaj, Department of Radiotherapy, Azien-

da Ospedaliero Universitaria Pisana, Via Roma 67, Pisa, Italy.

Email: delishaj@hotmail.com
}

doi: https://doi.org/10.14740/jocmr2936e tensity-modulated radiation therapy (IMRT) and particle radiation therapy, for the treatment of malignant brain tumors, it is possible to provide high absorbed doses to tumor tissue achieving good rates of tumor control [1-5]. However, the use of high dose radiation for the treatment of malignant brain tumors is typically at the expense of greater rates of radiation necrosis (RN) within the treatment volume. Additionally, stereotactic radiosurgery (SRS) achieves good control in metastatic brain tumors with the risk of $\mathrm{RN}$, especially in previously irradiated patients [6].

The major dose-limiting side effects of radiation therapy in the brain irradiation of patients with malignant brain tumors or brain metastases are RN, demyelination and vascular damage in the central nervous system (CNS) [7].

$\mathrm{RN}$ usually occurs three or more months after radiotherapy and the symptoms range from focal to generalized neurologic deficits, with a loss of patient autonomy and a negative impact on the quality of life [8].

The total radiation dose and volume of irradiated area, as well as the fractionation regimen are recognized as the main predictive factors of this severe sequela [9-12].

The pathogenesis is associated to endothelial cell dysfunction with an increased release of vascular endothelial growth factor (VEGF) and a subsequent serious damage of the bloodbrain barrier as shown in animal model of RN.

Deregulation of VEGF levels causes vascular permeability, brain edema, neuronal demyelination and finally, necrosis $[13,14]$.

Despite the difficulty of discriminating between tumor recurrence and radiation-induced tissue damage, magnetic resonance imaging (MRI) and magnetic resonance spectroscopy are considered the gold standard for the diagnosis of $\mathrm{RN}$, showing a contrast-enhancing mass with brain white matter changes and an edema within or adjacent to the site of the original tumor [15].

One other non-invasive technique for the diagnosis of RN is amino acid positron emission tomography (PET) [16, 17].

Different therapeutic options exist for the treatment of $\mathrm{RN}$. Historically, RN has been treated with corticosteroid therapy with poor results and many side effects such as behavioral changes, altered sleep patterns and changes in appetite.

Alternatively, the surgical decompression of necrotic areas can provide a beneficial palliative effect, despite the radical removal of the entire necrotic process being difficult to obtain in most cases [18].

Moreover, hyperbaric oxygen, anticoagulation, antiplatelet antibodies, laser interstitial thermal therapy and high-dose 
vitamin $\mathrm{E}$ have all been employed, albeit with limited efficacy, in the treatment of RN [19-22].

Recently, bevacizumab (a humanized murine monoclonal antibody against VEGF) has been suggested as a new treatment modality for brain RN [23-44].

There are limited data in the literature regarding the use of bevacizumab as a treatment for $\mathrm{RN}$ and current data are limited to case reports, small case series and one 14-patient randomized double-blind study. The purpose of this review was to perform a comprehensive analysis of all reported cases using bevacizumab for the treatment of cerebral RN. The data were compiled in a manner that allowed for an aggregation of a larger cohort of patients from a variety of institutions and clinical settings than in previously published articles.

\section{Materials and Methods}

\section{Search strategy}

In September 2016, we conducted a comprehensive literature search of the following electronic databases: PubMed, Web of Science, Scopus and Cochrane Library. The databases were searched using a combination of keywords pertaining to the treatment with bevacizumab of brain $\mathrm{RN}$ following brain irradiation. The research for the review was made using a combination of the keywords "radiation necrosis", "radiotherapy" and "bevacizumab" and fields pertaining to article title, abstract and keywords.

\section{Study selection}

We included in this systematic review randomized trials, nonrandomized trials, prospective studies and retrospective studies including patients affected by brain RN and treated with bevacizumab. Single case reports and small case series were included in this study.

Studies were excluded if there were not diagnoses of $\mathrm{RN}$, the cohort studies reported details regarding only radiotherapy treatment or treatment outcomes with bevacizumab were not reported.

\section{Data extraction and analysis}

Data extraction was performed by one reviewer and following a review of these articles, another review of all the referenced sources was conducted by a second reviewer. Two reviewers independently reviewed the abstracts and full texts of all retrieved papers to select suitable articles for the review.

We obtained the following information from each report: author identification, year of publication, medical centre, study design characteristics, study population, number of patients, age, sex, diagnoses of RN, radiotherapy treatment (RT technique, total dose, and dose for fraction), time between RT treatment and bevacizumab administration, bevacizumab treatment (doses of bevacizumab, number of cycles, frequency and administration), clinical response, radiographic response, cortisone reduction, median follow-up and toxicities.

Different radiographic criteria were used among the studies to diagnose RN but in general, they included the appearance of contrast enhancement and vasogenic edema observed on MRI, magnetic resonance spectroscopy or amino acid PET images.

\section{Statistical analysis}

Prior to data gathering, an exploration phase of the data was carried out; categorical data were described by frequency and percentage, whereas continuous data by mean, median and range. If necessary, following data exploration, analysis and calculation of frequencies, median and range were performed, due to the description of the end-points of review.

Other variables analyzed included the method of diagnosis of RN and the percentage change in T1 contrast enhancement and T2/FLAIR signal abnormality post-bevacizumab treatment.

All analyses were performed using the SPSS 22 software technology.

\section{Results}

\section{Research results}

Our searches generated a total of 388 results and through a process of screening, 21 publications were selected for the review. Of the 367 studies excluded, 293 were excluded during the first process of screening, after title and abstract analysis; 134 were excluded due to duplicate data (same study in more than one database research), 179 were excluded because they did not represent trials regarding $\mathrm{RN}$ and 22 were excluded because consisted in review (21) and a letter to the editor (1).

Moreover, 31 studies were excluded following one process of full text reading, because they did not fulfil the inclusion criteria (14 studies were excluded because there were no diagnoses of cerebral $\mathrm{RN}$ and 17 were excluded because reported details concerned only radiotherapy treatment).

Finally, 21 studies fulfilled the inclusion criteria and were included in our review; among them, one consisted of a randomized double-blind placebo-controlled trial, 11 were case reports and the remaining nine consisted of retrospective studies and case series.

A flowchart of the systematic literature search process is shown in Figure 1.

\section{Patients and treatment characteristics}

Our research generated 21 studies and 125 cases with diagnoses of RN where bevacizumab had been administered for the treatment of RN.

The median age was 57 (range: 8 - 80 years). Six pediatric patients were included in the cohort studies examined. 


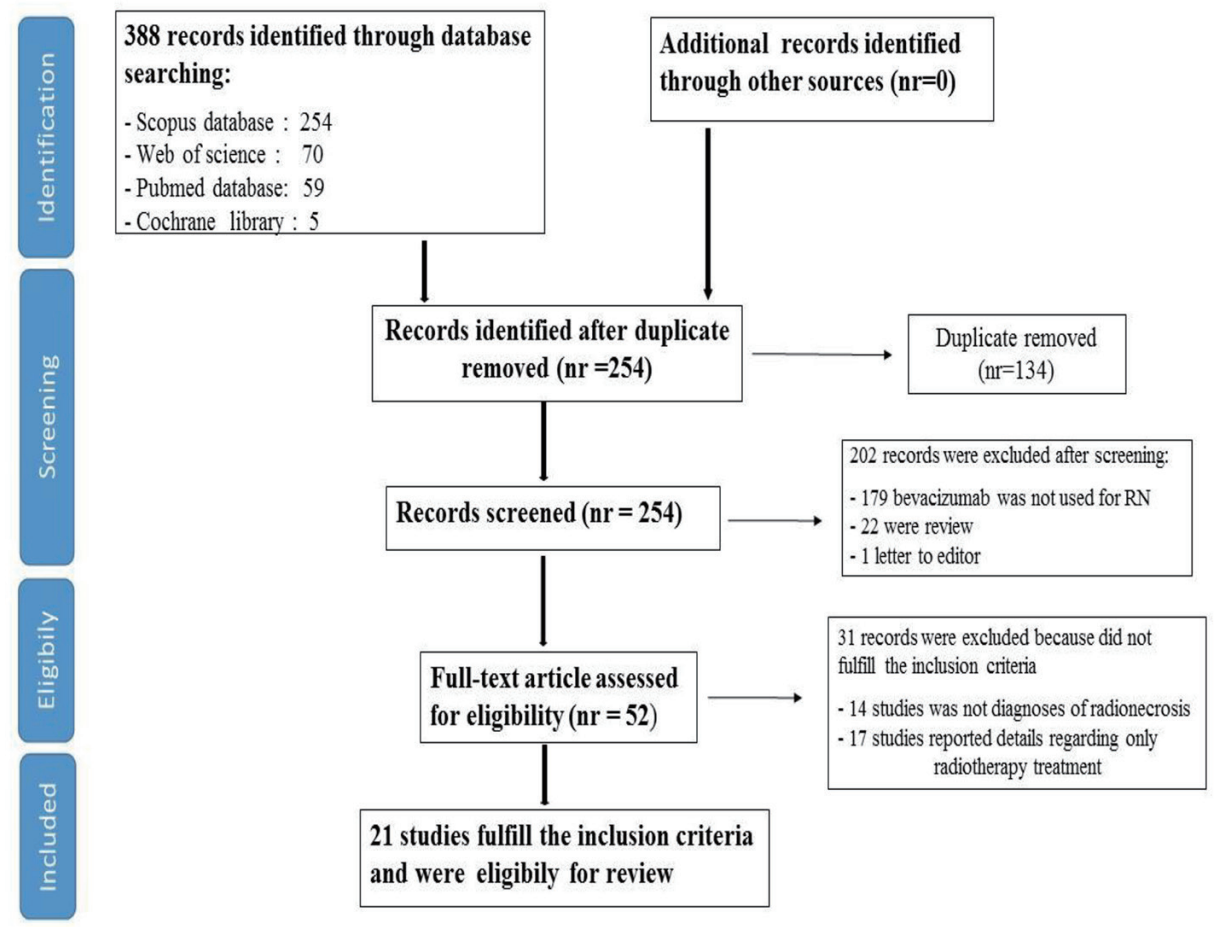

Figure 1. Flow chart of systematic literature search process.

At analyses, 63 patients (50.4\%) were male and 62 (49.6\%) were female (Table 1) [12, 25-44]. The most common tumors treated were metastatic brain tumors $(61,49 \%)$, glioblastoma $(28,22.3 \%)$, gliomas $(18,14.5 \%)$, meningiomas (seven, $6 \%)$, pontine glioma (three, $2.5 \%$ ), fibrous dysplasia of bone (one, $0.8 \%$ ), hemangiopericytoma (one, $0.8 \%$ ), malignant schwannoma (one, $0.8 \%$ ) and pituitary adenoma (one, $0.8 \%$ ). Three patients $(2.5 \%)$ were treated for arteriovenous malformations.

Regarding the conventional external beam radiation treatment, overall, 98 patients (78.4\%) underwent a first course radiotherapy treatment and the mean dosage was 58.9 Gy (range: 50 - $70 \mathrm{~Gy}$ ); of them, $28 \%$ of the cohort (42 patients) received a second course of radiotherapy.

Of the patients who underwent a second course of radiation therapy, $64 \%$ received radiosurgery.

Finally, 21\% (27 patients) of the cases received radiosurgery as the first course of treatment.

The most common sites of necrosis were the frontal lobe in 33 cases (27\%), temporal lobe in $24(19 \%)$, parietal lobe in $15(12 \%)$, brainstem in nine (7\%), occipital lobe in eight $(6 \%)$, thalamus in five $(4 \%)$, cerebellum in five $(4 \%)$ and basal ganglia in four $(3 \%)$. For 22 patients $(18 \%)$, the specified site of RN was not specified.

Of 125 patients treated, $17(14 \%)$ received a diagnosis of $\mathrm{RN}$ by tissue biopsy and the remaining $86 \%$ were diagnosed based on radiographic criteria.

The median time from the end of radiation treatment to bevacizumab treatment was 14 months (range: 1 - 180 months; Table 2) [12, 25-44].

Previous steroidal therapy for RN was reported in 17 $(80 \%)$ of 21 cohort studies and a reduction or stable dosage of steroidal therapy after bevacizumab treatment was observed in $97 \%$ of patients.

The median decrease in steroids following bevacizumab therapy was $8.6 \mathrm{mg}$ (range: 0 - $24 \mathrm{mg}$ ).

The median number of cycles of bevacizumab administered was four cycles and the median dosage was $7.5 \mathrm{mg} / \mathrm{kg}$ every 2 weeks (range from $5 \mathrm{mg} / \mathrm{kg}$ every 3 - 4 weeks to 15 $\mathrm{mg} / \mathrm{kg}$ every 3 weeks).

The median follow-up after bevacizumab treatment was 8 months (Table 2).

A clinical benefit with improvement of neurological symptoms after bevacizumab administration was observed in 114 patients (91.2\%). Seven patients (5.6\%) remained stable and four patients $(3.2 \%)$ have not had a clinical response after bevacizumab treatment alongside the progression of signs and symptoms following bevacizumab treatment.

Overall, 122 cases $(97.6 \%)$ had radiographic improvement after treatment with bevacizumab and only three patients (2.4\%) had radiographic progression of $\mathrm{RN}$ at first follow-up.

Pre- and post-treatment imaging decrease in T1 contrast enhancement and in T2/FLAIR signal abnormality was specifically described in nine $(42.8 \%)$ cohort studies. As shown in Table 2, the median decrease in $\mathrm{T} 1$ contrast enhancement and in T2/FLAIR signal abnormality was $64 \%$ and $60 \%$, respectively.

Globally, adverse events were reported in 14 of 21 studies analyzed (66\%) and the treatment of $\mathrm{RN}$ with a low dose of bevacizumab was well tolerated in all cohort studies analyzed; G3 adverse events were observed in only three cases (2.4\%) and consisted of pulmonary embolus.

Other adverse events included pulmonary embolus (four 


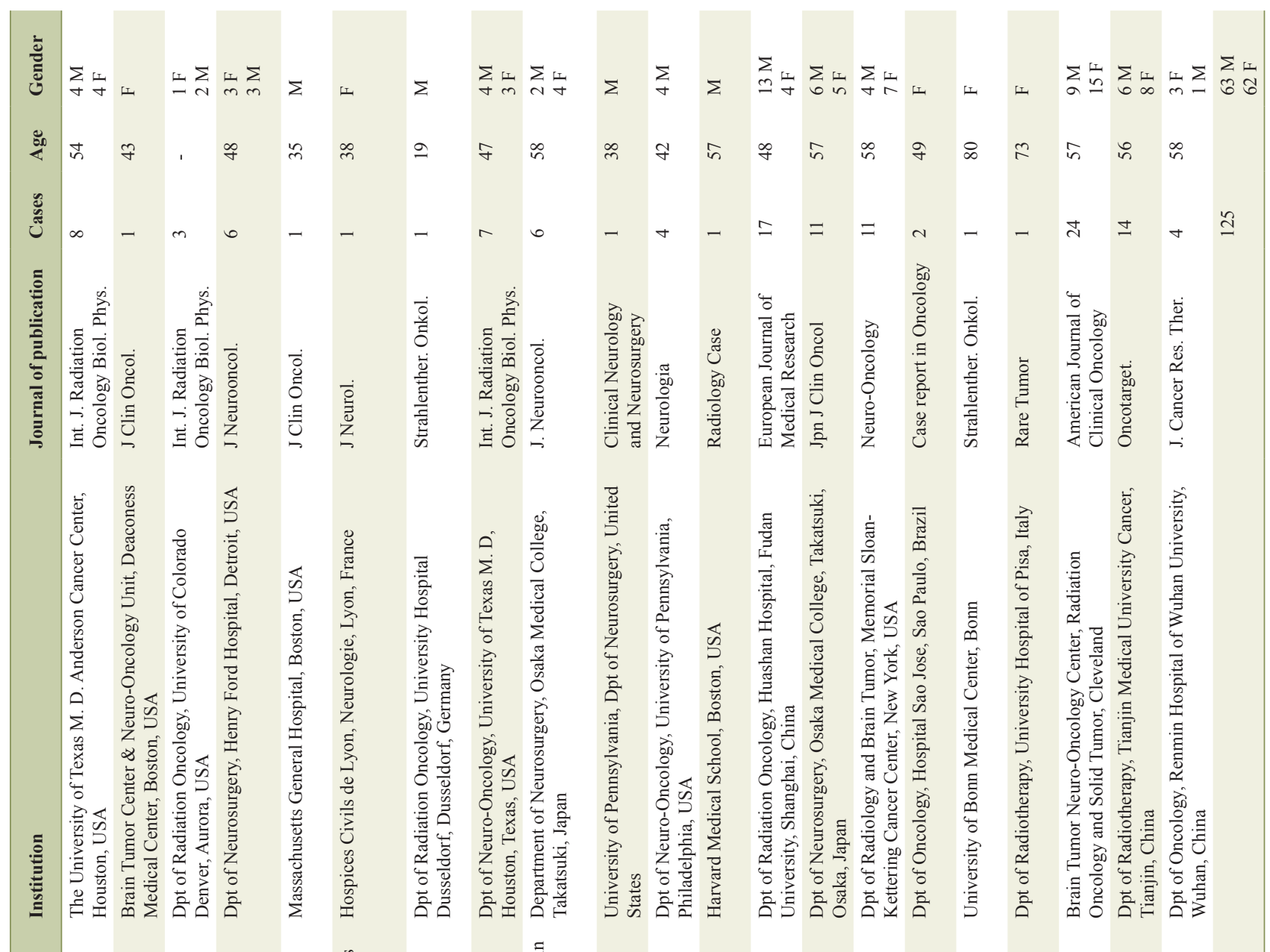

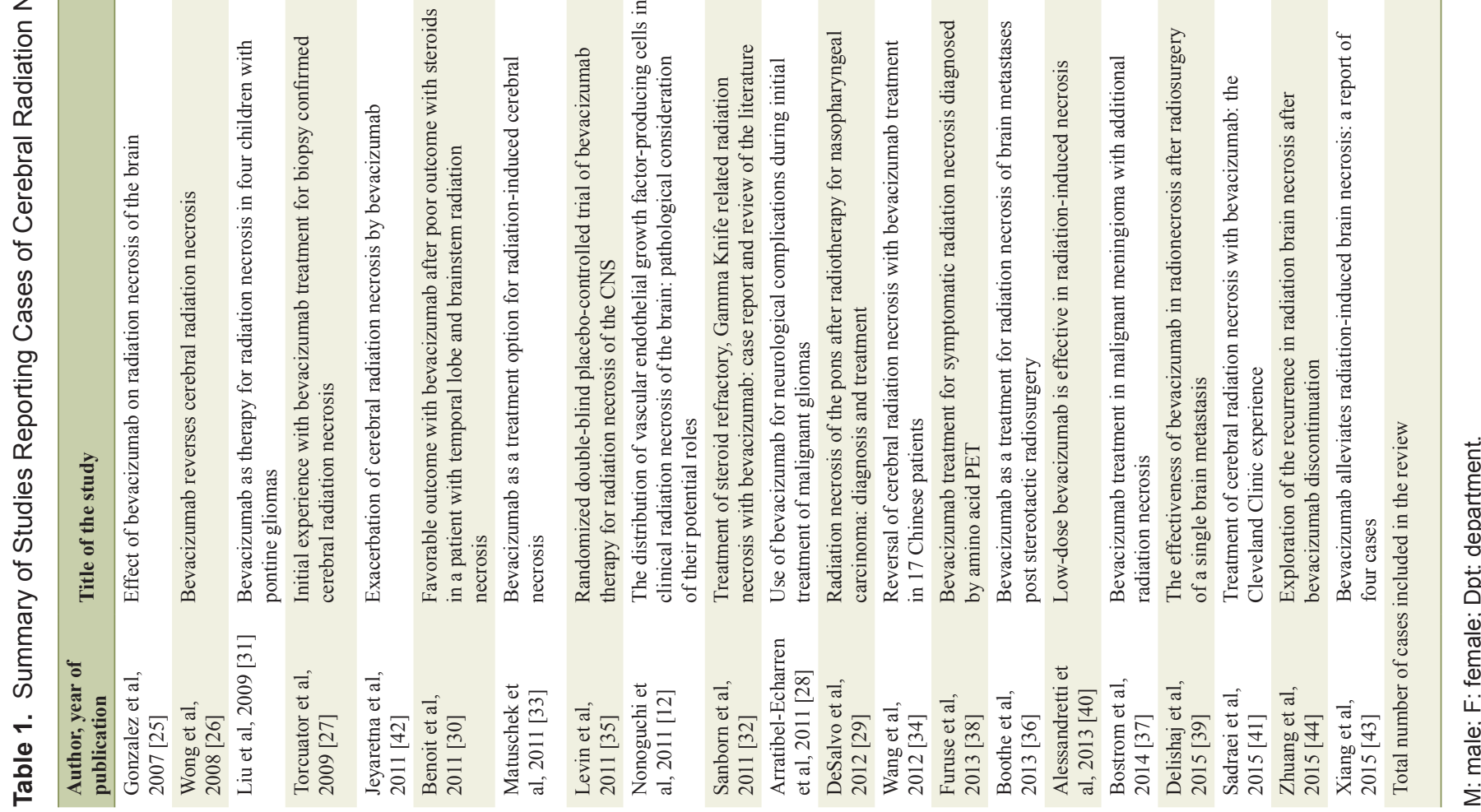



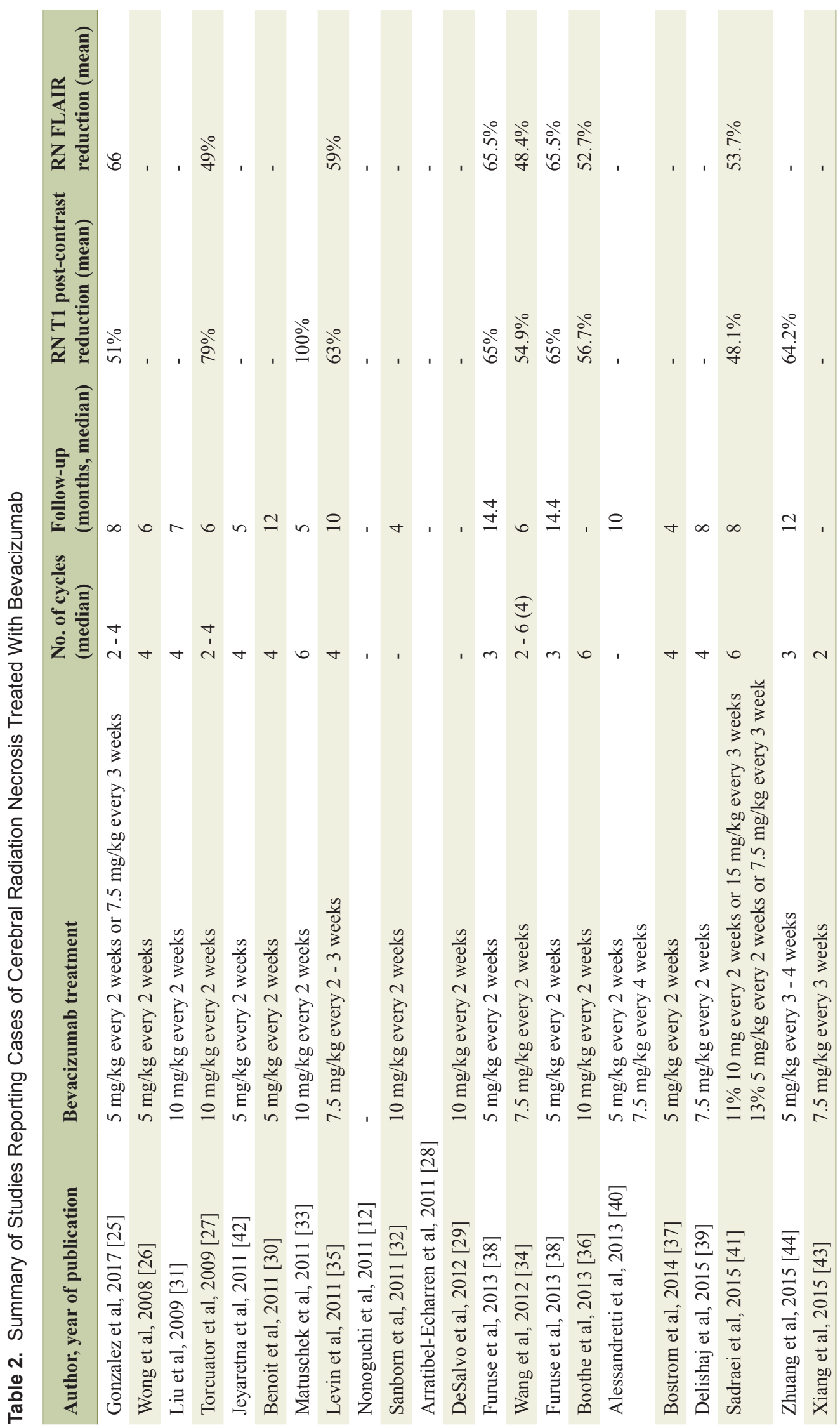
cases), hypertension (six cases), urinary tract infection (one case), fatigue (one case), proteinuria (one case), sagittal sinus thrombosis (one case), aspiration pneumonia (one case) and pneumonia with severe sepsis (one case).

Finally, three patients had small vessel thrombosis, which caused ischemic changes.

\section{Discussion}

$\mathrm{RN}$ of brain tissue is a possible late complication occurring after radiotherapy treatment and is usually diagnosed at followup imaging or at the appearance of neurological symptoms.

Different therapeutic options are available for the treatment of RN; historically, $\mathrm{RN}$ has been treated with corticosteroid therapy with poor results and many side effects, such as behavioral changes, altered sleep patterns and changes in appetite.

Alternatively, the surgical decompression of a necrotic area can provide a beneficial palliative effect [18]. Moreover, hyperbaric oxygen, anticoagulation, antiplatelet antibodies, laser interstitial thermal therapy and high-dose vitamin $\mathrm{E}$ have all been used, albeit with limited efficacy, in the treatment of RN [19-22].

Recently, bevacizumab (a humanized murine monoclonal antibody against VEGF) has been suggested as a new treatment modality for brain RN.

The rationale of using bevacizumab in patients with $\mathrm{RN}$ relies on higher VEGF levels in areas of hypoxia and necrosis due to endothelial cell dysfunction and a subsequent serious damage of the blood-brain barrier as shown in animal model of RN $[13,14]$. Hypoxia-inducible factor-1 (HIF-1), a heterodimeric transcription factor composed of alpha and beta subunits, is an important regulator of angiogenesis through the activation of pro-angiogenic factors, among which VEGF [22]. In normoxia, the alpha subunit of HIF-1 is rapidly degraded, while it remains stable in hypoxic conditions; the nondegradation of HIF-1 alpha could lead to the over-expression of VEGF. As a matter of fact, the highest levels of VEGF expression have been observed in necrotic or hypoxic areas [24]. These preclinical considerations suggest that the use of bevacizumab in patients with $\mathrm{RN}$ may have a benefit improving in clinical and radiographic evaluation with reduction of RN.

Despite poor data in the existing literature, bevacizumab treatment appears to be clinical beneficial for patients affected by post-RN lesions [25-44].

Gonzalez et al [25], in a retrospective analysis published in 2007, demonstrated an improvement in neurological symptoms, as well as radiographic improvement, in eight patients affected by radiation brain necrosis after treatment with bevacizumab at a dose of $5 \mathrm{mg} / \mathrm{kg}$ every 2 weeks, or $7.5 \mathrm{mg} / \mathrm{kg}$ every 3 weeks. The average area change in the T1-weighted post-Gd-contrast abnormalities reduction was $48 \%$ and in FLAIR images $60 \%$, respectively.

Torcuator et al [27] presented the data of six patients with biopsy-proven RN treated with low doses of bevacizumab. All patients showed radiographic response and an average reduction of $79 \%$ for the T1 post-gadolinium studies and $49 \%$ for the FLAIR images.
Moreover, Wang et al [34] demonstrated bevacizumab at a dose of $7.5 \mathrm{mg} / \mathrm{kg}$ every 2 weeks to be effective in the reduction of cerebral edema, at radiographic evaluation, with a subsequent improvement of clinical symptoms in 17 patients affected by a symptomatic brain RN.

Levin et al [35], in a randomized placebo-controlled trial, reported the efficacy of bevacizumab at the dose of $7.5 \mathrm{mg} / \mathrm{kg}$ every 3 weeks for both reducing the necrotic area and improving clinical symptoms in 14 patients with biopsy-proven RN.

Finally, Sadraei et al [41] published the data of 24 patients affected by RN and treated with low doses of bevacizumab, with a post-treatment MRI radiographic improvement in 23 of 24 patients. The average change in the T1-weighted post-contrast MRI was a decrease of $48.1 \%$ and the average change in the fluid-attenuated inversion-recovery images was a decrease of $53.7 \%$. There, mean daily dose reduction of dexamethasone was $9.4 \mathrm{mg}$ and the treatment with bevacizumab was well tolerated, with only one grade 3 adverse event. Similar results were reported by other authors [26, 28-33, 36-40, 42-44] in their retrospective studies.

The inherent limitations of our study were due to the retrospective studies analyzed, the small number of patients reported in the studies, the patients having been treated for different conditions, different radiation doses, different radiation modalities and with limited follow-up after bevacizumab therapy. Furthermore, the diagnosis of $\mathrm{RN}$ was made by radiologic evaluation in the majority of the studies analyzed in this review and the patients were treated in different institutions and countries.

Furthermore, there is a publication bias present, because only patients who responded to bevacizumab were likely to be included in the published literature.

Overall, based on our results of the review conducted, a low dose of bevacizumab appears to be efficacious for reducing $\mathrm{RN}$ in patients affected by brain metastases and $\mathrm{RN}$ after radiotherapy treatment, seemingly without any risk of clinical complications.

\section{Conclusions}

Based on the data of our review, low-dose bevacizumab appears to be a promising agent for the treatment of brain $\mathrm{RN}$, as well as an anti-cancer drug.

Currently, the optimal scheduling and treatment duration has not yet been established.

Nevertheless, a significant amount of recurrence has been described several months after bevacizumab discontinuation, indicating that repeated cycles may be warranted in the absence of contraindications.

Future prospective studies are required to properly evaluate the role of bevacizumab as an efficacious agent against post-RN as well as to define the optimal scheduling, dosage and duration of therapy.

\section{Conflicts of Interest}

The authors declare no conflicts of interest. 


\section{References}

1. Fitzek MM, Thornton AF, Rabinov JD, Lev MH, Pardo FS, Munzenrider JE, Okunieff P, et al. Accelerated fractionated proton/photon irradiation to 90 cobalt gray equivalent for glioblastoma multiforme: results of a phase II prospective trial. J Neurosurg. 1999;91(2):251-260.

2. Iuchi T, Hatano K, Narita Y, Kodama T, Yamaki T, Osato K. Hypofractionated high-dose irradiation for the treatment of malignant astrocytomas using simultaneous integrated boost technique by IMRT. Int J Radiat Oncol Biol Phys. 2006;64(5):1317-1324.

3. Kawabata S, Miyatake S, Kuroiwa T, Yokoyama K, Doi A, Iida K, Miyata S, et al. Boron neutron capture therapy for newly diagnosed glioblastoma. J Radiat Res. 2009;50(1):51-60.

4. Miyatake S, Kawabata S, Kajimoto Y, Aoki A, Yokoyama K, Yamada M, Kuroiwa T, et al. Modified boron neutron capture therapy for malignant gliomas performed using epithermal neutron and two boron compounds with different accumulation mechanisms: an efficacy study based on findings on neuroimages. J Neurosurg. 2005;103(6):1000-1009.

5. Tanaka M, Ino Y, Nakagawa K, Tago M, Todo T. Highdose conformal radiotherapy for supratentorial malignant glioma: a historical comparison. Lancet Oncol. 2005;6(12):953-960.

6. Ohguri T, Imada H, Kohshi K, Kakeda S, Ohnari N, Morioka T, Nakano K, et al. Effect of prophylactic hyperbaric oxygen treatment for radiation-induced brain injury after stereotactic radiosurgery of brain metastases. Int $\mathbf{J}$ Radiat Oncol Biol Phys. 2007;67(1):248-255.

7. Marks JE, Wong J. The risk of cerebral radionecrosis in relation to dose, time and fractionation. A follow-up study. Prog Exp Tumor Res. 1985;29:210-218.

8. Ruben JD, Dally M, Bailey M, Smith R, McLean CA, Fedele P. Cerebral radiation necrosis: incidence, outcomes, and risk factors with emphasis on radiation parameters and chemotherapy. Int J Radiat Oncol Biol Phys. 2006;65(2):499-508.

9. Wara WM, Bauman GS, Sneed PK. Brain, brain stem and cerebellum. In: Perez CA, Brady LW: Principles and Practice of Radiation Oncology, 3rd ed. Philadelphia, PA: Lippincott-Raven press; 1998: p. 777-828. 121.

10. Li YQ, Ballinger JR, Nordal RA, Su ZF, Wong CS. Hypoxia in radiation-induced blood-spinal cord barrier breakdown. Cancer Res. 2001;61(8):3348-3354.

11. Burger PC, Boyko OB. The pathology of central nervous system radiation injury. In: Gutin PH, Leibel SA, Sheline GE, (Eds.): Radiation injury of the nervous system. New York: Raven Press, Ltd.; 1991; p. 191-208.

12. Nonoguchi N, Miyatake S, Fukumoto M, Furuse M, Hiramatsu R, Kawabata S, Kuroiwa T, et al. The distribution of vascular endothelial growth factor-producing cells in clinical radiation necrosis of the brain: pathological consideration of their potential roles. J Neurooncol. 2011;105(2):423-431.

13. Wong ET, Brem S. Antiangiogenesis treatment for glio- blastoma multiforme: challenges and opportunities. J Natl Compr Canc Netw. 2008;6(5):515-522.

14. Kim JH, Chung YG, Kim CY, Kim HK, Lee HK. Upregulation of VEGF and FGF2 in normal rat brain after experimental intraoperative radiation therapy. J Korean Med Sci. 2004;19(6):879-886.

15. Gerstner ER, Chen PJ, Wen PY, Jain RK, Batchelor TT, Sorensen G. Infiltrative patterns of glioblastoma spread detected via diffusion MRI after treatment with cediranib. Neuro Oncol. 2010;12(5):466-472.

16. Imahori Y, Ueda S, Ohmori Y, Kusuki T, Ono K, Fujii R, Ido T. Fluorine-18-labeled fluoroboronophenylalanine PET in patients with glioma. J Nucl Med. 1998;39(2):325333.

17. Takahashi Y, Imahori Y, Mineura K. Prognostic and therapeutic indicator of fluoroboronophenylalanine positron emission tomography in patients with gliomas. Clin Cancer Res. 2003;9(16 Pt 1):5888-5895.

18. McPherson CM, Warnick RE. Results of contemporary surgical management of radiation necrosis using frameless stereotaxis and intraoperative magnetic resonance imaging. J Neurooncol. 2004;68(1):41-47.

19. Chan AS, Cheung MC, Law SC, Chan JH. Phase II study of alpha-tocopherol in improving the cognitive function of patients with temporal lobe radionecrosis. Cancer. 2004;100(2):398-404.

20. Chuba PJ, Aronin P, Bhambhani K, Eichenhorn M, Zamarano L, Cianci P, Muhlbauer M, et al. Hyperbaric oxygen therapy for radiation-induced brain injury in children. Cancer. 1997;80(10):2005-2012.

21. Glantz MJ, Burger PC, Friedman AH, Radtke RA, Massey EW, Schold SC, Jr. Treatment of radiation-induced nervous system injury with heparin and warfarin. Neurology. 1994;44(11):2020-2027.

22. Forsythe JA, Jiang BH, Iyer NV, Agani F, Leung SW, Koos RD, Semenza GL. Activation of vascular endothelial growth factor gene transcription by hypoxia-inducible factor 1. Mol Cell Biol. 1996;16(9):4604-4613.

23. Furuse M, Kawabata S, Kuroiwa T, Miyatake S. Repeated treatments with bevacizumab for recurrent radiation necrosis in patients with malignant brain tumors: a report of 2 cases. J Neurooncol. 2011;102(3):471-475.

24. Tye K, Engelhard HH, Slavin KV, Nicholas MK, Chmura SJ, Kwok Y, Ho DS, et al. An analysis of radiation necrosis of the central nervous system treated with bevacizumab. J Neurooncol. 2014;117(2):321-327.

25. Gonzalez J, Kumar AJ, Conrad CA, Levin VA. Effect of bevacizumab on radiation necrosis of the brain. Int $\mathrm{J}$ Radiat Oncol Biol Phys. 2007;67(2):323-326.

26. Wong ET, Huberman M, Lu XQ, Mahadevan A. Bevacizumab reverses cerebral radiation necrosis. J Clin Oncol. 2008;26(34):5649-5650.

27. Torcuator R, Zuniga R, Mohan YS, Rock J, Doyle T, Anderson J, Gutierrez J, et al. Initial experience with bevacizumab treatment for biopsy confirmed cerebral radiation necrosis. J Neurooncol. 2009;94(1):63-68.

28. Arratibel-Echarren I, Albright K, Dalmau J, Rosenfeld MR. Use of Bevacizumab for neurological complications during initial treatment of malignant gliomas. Neurolo- 
gia. 2011;26(2):74-80.

29. DeSalvo MN. Radiation necrosis of the pons after radiotherapy for nasopharyngeal carcinoma: diagnosis and treatment. J Radiol Case Rep. 2012;6(7):9-16.

30. Benoit A, Ducray F, Cartalat-Carel S, Psimaras D, Ricard D, Honnorat J. Favorable outcome with bevacizumab after poor outcome with steroids in a patient with temporal lobe and brainstem radiation necrosis. J Neurol. 2011;258(2):328-329.

31. Liu AK, Macy ME, Foreman NK. Bevacizumab as therapy for radiation necrosis in four children with pontine gliomas. Int J Radiat Oncol Biol Phys. 2009;75(4):11481154.

32. Sanborn MR, Danish SF, Rosenfeld MR, O'Rourke D, Lee JY. Treatment of steroid refractory, Gamma Knife related radiation necrosis with bevacizumab: case report and review of the literature. Clin Neurol Neurosurg. 2011;113(9):798-802.

33. Matuschek C, Bolke E, Nawatny J, Hoffmann TK, Peiper $\mathrm{M}$, Orth K, Gerber PA, et al. Bevacizumab as a treatment option for radiation-induced cerebral necrosis. Strahlenther Onkol. 2011;187(2):135-139.

34. Wang Y, Pan L, Sheng X, Mao Y, Yao Y, Wang E, Zhang $\mathrm{N}$, et al. Reversal of cerebral radiation necrosis with bevacizumab treatment in 17 Chinese patients. Eur J Med Res. 2012;17:25.

35. Levin VA, Bidaut L, Hou P, Kumar AJ, Wefel JS, Bekele $\mathrm{BN}$, Grewal J, et al. Randomized double-blind placebocontrolled trial of bevacizumab therapy for radiation necrosis of the central nervous system. Int J Radiat Oncol Biol Phys. 2011;79(5):1487-1495.

36. Boothe D, Young R, Yamada Y, Prager A, Chan T, Beal $\mathrm{K}$. Bevacizumab as a treatment for radiation necrosis of brain metastases post stereotactic radiosurgery. Neuro
Oncol. 2013;15(9):1257-1263.

37. Bostrom JP, Seifert M, Greschus S, Schafer N, Glas M, Lammering G, Herrlinger U. Bevacizumab treatment in malignant meningioma with additional radiation necrosis. An MRI diffusion and perfusion case study. Strahlenther Onkol. 2014;190(4):416-421.

38. Furuse M, Nonoguchi N, Kawabata S, Yoritsune E, Takahashi M, Inomata T, Kuroiwa T, et al. Bevacizumab treatment for symptomatic radiation necrosis diagnosed by amino acid PET. Jpn J Clin Oncol. 2013;43(3):337341.

39. Delishaj D, Ursino S, Pasqualetti F, Pesaresi I, Desideri I, Cosottini M, Laliscia C, et al. The Effectiveness of Bevacizumab in Radionecrosis After Radiosurgery of a Single Brain Metastasis. Rare Tumors. 2015;7(4):6018.

40. Alessandretti M, Buzaid AC, Brandao R, Brandao EP. Low-dose bevacizumab is effective in radiation-induced necrosis. Case Rep Oncol. 2013;6(3):598-601.

41. Sadraei NH, Dahiya S, Chao ST, Murphy ES, OseiBoateng K, Xie H, Suh JH, et al. Treatment of cerebral radiation necrosis with bevacizumab: the Cleveland clinic experience. Am J Clin Oncol. 2015;38(3):304-310.

42. Jeyaretna DS, Curry WT, Jr., Batchelor TT, StemmerRachamimov A, Plotkin SR. Exacerbation of cerebral radiation necrosis by bevacizumab. J Clin Oncol. 2011;29(7):e159-162.

43. Xiang-Pan L, Yuxin C, Xiao-Fei W, Na L, Tang-Peng X, Xiao-Tao X, Chang-Li R, et al. Bevacizumab alleviates radiation-induced brain necrosis: A report of four cases. $\mathrm{J}$ Cancer Res Ther. 2015;11(2):485-487.

44. Zhuang H, Yuan X, Chang JY, Song Y, Wang J, Yuan Z, Wang $X$, et al. Exploration of the recurrence in radiation brain necrosis after bevacizumab discontinuation. Oncotarget. 2016;7(30):48842-48849. 